\title{
Entrelacs
}

Cinéma et audiovisuel

$10 \mid 2013$

Le Toucher

\section{Rêve et matière. Toucher-coller dans les films de Gunvor Nelson}

Julie Savelli

\section{(2) OpenEdition}

Journals

Édition électronique

URL : http://journals.openedition.org/entrelacs/525

DOI : 10.4000/entrelacs.525

ISSN : 2261-5482

Éditeur

Éditions Téraèdre

Référence électronique

Julie Savelli, «RÊVE ET MATIÈRE. TOUCHER-Coller DANS LeS films DE GUNVOR NELSON », Entrelacs

[En ligne], 10 | 2013, mis en ligne le 12 septembre 2013, consulté le 19 avril 2019. URL : http://

journals.openedition.org/entrelacs/525; DOI : 10.4000/entrelacs.525

Ce document a été généré automatiquement le 19 avril 2019.

Tous droits réservés 


\title{
Rêve et matière. Toucher-coller dans les films de Gunvor Nelson
}

\author{
Julie Savelli
}

"I just pass my hands over the surface of things. " ${ }^{1}$

Gunvor Nelson

1 L'artiste américano-suédoise Gunvor Nelson a réalisé vingt-six films de longueurs variées ${ }^{2}$ depuis 1966. De la pellicule au numérique, pour la salle et les musées, elle se plaît à expérimenter le matériau de la réalité par les moyens du cinéma sous une forme personnelle et poétique. Cette œuvre déterminante a fait l'objet de prestigieuses rétrospectives en Europe et aux États-Unis - notamment au MoMA à New York en 2006 mais elle n'en demeure pas moins secrète, dans la tradition du cinéma underground. Si le travail de Gunvor Nelson doit nécessairement être replacé dans le contexte historique et esthétique du cinéma expérimental américain auquel il appartient ${ }^{3}$ tant par ses thématiques (le corps, la mémoire, la femme...) que par sa recherche formelle, ce n'est pas ici notre objet. Cet essai s'intéresse au rêve matériel qui anime la conscience créatrice de la cinéaste dans le temps de la mise en œuvre.

2 Gunvor Nelson est cinéaste, peintre et photographe. Dans tous ses films elle porte une attention particulière à la matière, au matériel et à la matérialité. La transformation explicite du matériau (qu'il s'agisse d'un corps, d'une peinture ou d'un paysage) par une expérimentation proprement cinématographique (prise de vue et de son, lumière, développement, montage, etc.) engage ce cinéma au-delà de son accomplissement même, le rattachant à cette autre histoire du cinéma, à l'écart de la production dite dominante. C'est un cinéma dont l'objectif premier n'est pas de servir la narration ou la représentation mais plutôt la creative method ${ }^{4}$, donnant explicitement à voir l'élaboration du film.

3 Gunvor Nelson explore le monde comme elle le perçoit et le rêve, tel qu'elle le ressent et le pense, privilégiant pour ce faire le temps matériel de la fabrication de l'œuvre. Elle déroge aux attentes formatées par les besoins de l'industrie cinématographique pour restituer l'empreinte de son être et de sa subjectivité dans l'immédiateté du film à faire. 
La cinéaste refuse donc cette " avance $»^{5}$ sur le film en gestation qui la conduirait à porter moins d'attention au présent si elle se consacrait à l'anticipation composite d'un projet écrit. Elle tente au contraire de saisir l'histoire de l'œuvre au moment où elle se réalise, de penser l'émergence sensible du film, écrivant le cinéma dans le temps explicite de sa création. Pour cela, elle va droit au film : on ne trouvera pas de scénario ${ }^{6}$ ou de découpage en amont du tournage mais plutôt, selon les projets, des états provisoires qui contiennent une infinité d'échappées en accord avec le trajet de l'œuvre à venir.

Gunvor Nelson ne cherche pas à contraindre le matériau ${ }^{7}$, ni celui de la réalité ni celui du cinéma ; elle travaille au contraire en interaction avec lui pour essayer de créer une vibration spécifique et rendre compte d'une expérience purement matérielle. C'est dire de manière manifeste que la création n'est pas une forme abstraite, une chose purement mentale coupée de la pratique mais qu'elle se confond bien avec le poïein, le faire. Ainsi la cinéaste a recours, sous différentes formes, à la technique du collage car ce mode d'expression lui permet d'avoir un contact physique avec le matériau : les doigts entrent directement en relation avec la matière de, et dans, l'image. Le collage consiste pour Gunvor Nelson en un recouvrement de l'image par l'image. Peu importe le procédé utilisé (animation directe, montage cut, surimpression, mixage son, etc.) ou le support recouvert à l'écran - dessin, peinture, photographie, papier, journal, carte postale -, les couches se superposent sous nos yeux comme dans un rêve éveillé, et la réalité devient autre. Cette manipulation de la matière et du matériel place le toucher au cœur des films, nourrissant la question haptique ${ }^{8}$ à l'écran. Les qualités tactiles ne sont pas seulement liées ici à la représentation de la main ou du collage mais elles dépendent aussi de l'implication du corps des spectateurs dans le matériau filmique. Le regard prend alors le relais du toucher pour éprouver la création "à vue ", nous engageant simultanément dans le faire et le ressentir de l'œuvre. ${ }^{9}$

5 Le collage a une fonction génétique dans les films de Gunvor Nelson. Son cinéma s'éclaire par les processus de son propre travail, devenant le phénomène significatif de la conscience créatrice de la cinéaste. Dans le prolongement de La boite verte de Marcel Duchamp ${ }^{10}$, l'attention ne porte plus sur l'œuvre achevée mais sur l'idée matérielle qui a permis son accomplissement, comme pour tenter de ressentir et de comprendre d'où elle vient.

6 Nous proposons d'envisager trois formes de «toucher-coller » tel que le pratique Gunvor Nelson à différentes périodes de son œuvre. Dans les premiers films expérimentaux tournés en $16 \mathrm{~mm}$ à la fin des années soixante, le collage est une pratique manuelle qui véhicule une pensée sensible et personnelle. Pour penser, et faire penser, la cinéaste a recours au choc perceptif propre à l'agencement disparate des images et des sons, produisant ainsi un dialogue matériel qui participe à une nouvelle architecture du monde. Dans la série de films réalisée entre 1983 et 1988 au Filmworkshop de Stockholm, le corps de la cinéaste se mêle à la matière travaillée devenant un " corps-monde ", à la fois éprouvé et fantasmé. Les films sont l'expression d'une rencontre entre les mains et les choses. Enfin, nous interrogerons l'imaginaire du matériau que le collage véhicule dans des travaux plus récents tournés en vidéo. Le rêve matériel, reflet d'une technique alors nouvelle, implique ici les spectateurs dans une expérience d'inspiration impressionniste. 


\section{Le collage sensible de la pensée}

7 Claude Lévi-Strauss définit la "pensée sauvage » ou "mythique »11 à la fois par une dévorante ambition symbolique et par une attention scrupuleuse entièrement tournée vers le concret. Cette pensée se distingue en ce qu'elle cherche à saisir le monde selon des combinaisons variées où chaque élément, autonome, existe pour lui-même sans être complètement absorbé par le tout. Elle classifie, multiplie les catégories et les oppose, établissant des règles de transformation de l'une à l'autre pour appréhender l'univers.

Les premiers collages expérimentaux réalisés à la fin des années soixante, semblent animés par une forme sauvage de la pensée. Elle «bricole»: les images, les sons sont autant de fragments disparates mis en dialogue selon un système personnel de signification. Sur le principe du patchwork, elle assemble des morceaux de son histoire personnelle (dessins, peintures, photographies, cartes postales, prises de vue et de son réelles) pour leur (ré)découvrir du sens indépendamment de leurs ensembles respectifs tels les chiffons de Walter Benjamin ${ }^{12}$. Ce quotidien, qu'il soit familial, social ou culturel, devient le matériau concret d'un travail exploratoire. La cinéaste crée de nouvelles correspondances qui participent à une compréhension matérielle de la réalité. Gunvor Nelson creuse son environnement familier et cherche à mettre en valeur son envers, sa complexité. Les fragments ordinaires se voient ainsi renouvelés par le collage sensible de la pensée.

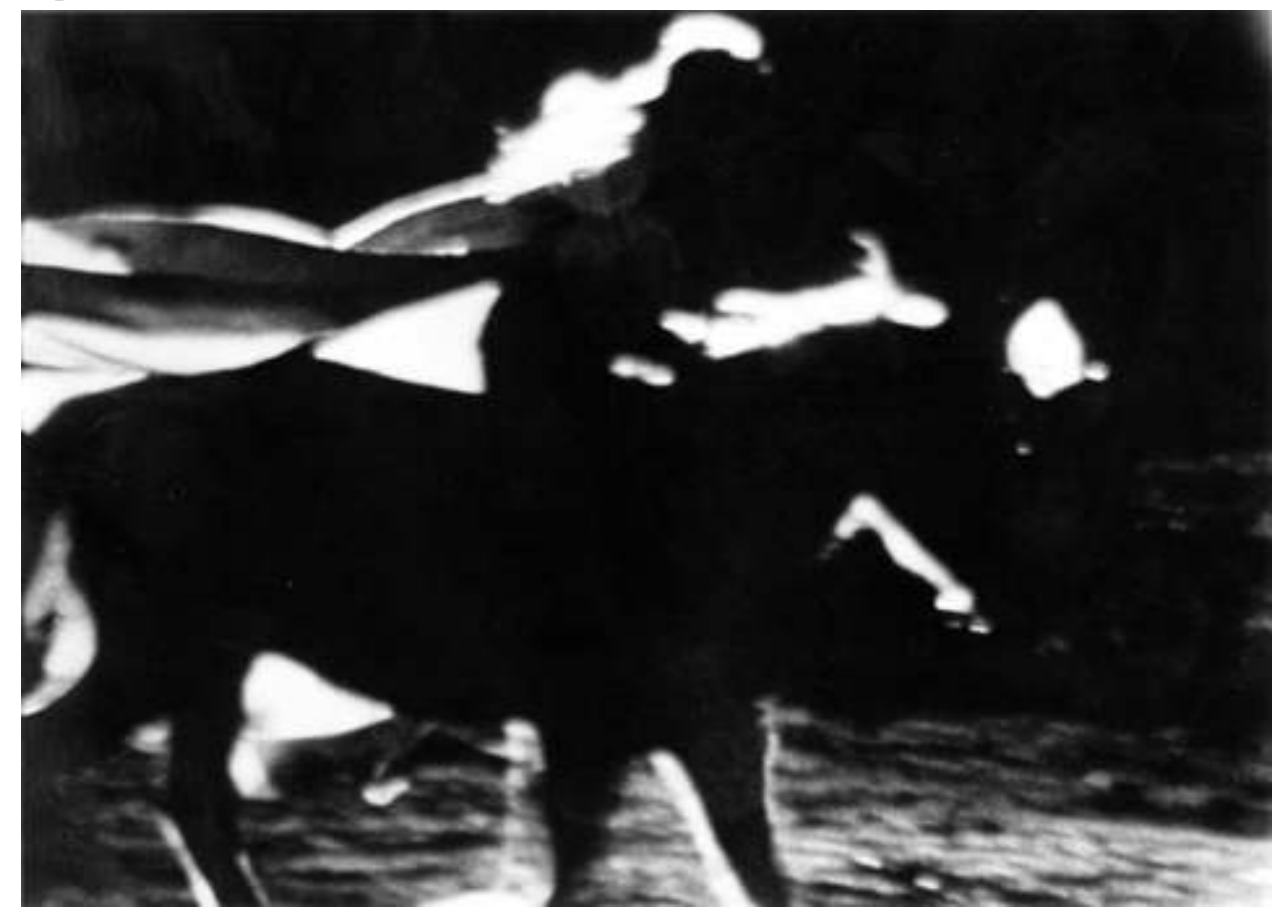

FIgURE 1 : MY NAME IS OONA, 1969, 16MM, 10 MIN

9 Gunvor Nelson soulève une réalité sociale très concrète dans son premier film, Schmeerguntz (Nelson \& Wiley, 1966, 15', 16 mm). La cinéaste a conscience de vivre en cinéma et interroge, sous forme de clins d'œil, de citations, de détournements, la place qu'elle occupe en tant que femme dans la société américaine. Cette pratique du collage recouvre à la fois une dimension autobiographique et contestataire. Gunvor Nelson pense sa relation au monde à la fois de son point de vue de jeune femme, européenne et 
émancipée, et d'un point de vue féministe. Le personnel et le politique cohabitent avec humour au moyen d'un découpage d'images de différentes natures qui, une fois assemblées, dialoguent sur la base d'oppositions et de combinaisons diverses. La cinéaste, et sa co-réalisatrice Dorothy Wiley, proposent une réflexion sur l'Amérique puritaine à partir d'un montage d'images télévisées (actualités, feuilletons, jeux), de publicités découpées dans des magazines féminins et de plans crus tournés en noir et blanc dont elles sont les interprètes - une femme vomit, enlève son tampon, nettoie les fesses sales d'un nourrisson, récure un évier... Ce puzzle sans concession tourne la société des années soixante en dérision (même si la plupart des images télévisées proviennent pourtant d'un stock de pellicule qui date de la décennie précédente). Par leur travail de coupe, de colle et d'animation, les deux jeunes cinéastes repensent les images collectives de la société médiatique pour mettre en lumière un décalage avec le quotidien de la femme dénonçant ainsi un sexisme latent.

Puisant à nouveau dans son univers familier, Gunvor Nelson prolonge sa réflexion sur l'expérience féminine à travers le portrait court et intense de sa petite fille, Oona, alors âgée d'une dizaine d'années. ${ }^{13}$ My Name is Oona $(1969,10$ ', 16 mm) s'ouvre sur le visage riant de l'enfant filmée en noir et blanc. La mosaïque de la pensée s'opère essentiellement par le jeu de superposition et de surimpression des images qui crée d'autres images par transparence. La cinéaste peint un double portrait, physique et psychique, révèlant la puissance primitive d'Oona sur le mode du palimpseste. Les longs cheveux blonds de la petite amazone se confondent avec la crinière de l'animal qu'elle chevauche au ralenti. Le visage de l'enfant devient paysage. Le grain de sa peau se mêle à celui de la pellicule. Cette métamorphose est achevée par le traitement de la voix, d'abord légèrement réverbérée, puis violemment distordue. À la manière d'un jeu, Oona répète inlassablement son prénom, égrène les jours de la semaine. La fillette ressent un plaisir certain dans le rythme de l'énonciation mais la répétition lancinante renvoie aussi aux difficultés de l'apprentissage et à la fonction autoritaire du langage. Gunvor Nelson cherche à trouver d'autres correspondances pour dire la complexité du monde, pour défendre un rapport libre, vivant, un rapport organique d'éloignement et de proximité entre l'image et le son. Cette libération audio-visuelle ${ }^{14} \mathrm{du}$ récit est essentielle - et traverse plus largement toute l'œuvre de la cinéaste. Petit être constamment en mouvement, Oona occupe instinctivement l'espace argentique : une chorégraphie invraisemblable se déploie dans une série de fragments brefs nous faisant éprouver l'agitation de ce corps d'enfant de l'intérieur en même temps que du point de vue maternel. Galopant avec grâce et assurance, Oona en vient à transcender sa propre existence par l'évocation des cavalières de la mythologie nordique. Et le film se clôt sur un chant scandinave, peut-être celui de la mère-cinéaste hors champ, vers laquelle l'enfant tend son visage attentif. My Name is Oona est un film de montage qui nous fait sentir à la fois l'écoulement du temps et sa discontinuité. Les images et les sons sont autant de blocs de mouvement-durée qui explorent l'éternelle présence de l'enfance révolue.

Gunvor Nelson se réapproprie le monde sous une forme primitive à travers un «bric à brac» d'images et de sons, traces infimes d'événements extraits d'une histoire personnelle. En assemblant des fragments disparates, la cinéaste leur confère une autre nature, manifestant en eux, par l'acte de voir et de faire voir, un caractère ontologique ${ }^{15}$. Chaque détail concret du quotidien est une révélation qui participe à la dimension photogénique ${ }^{16}$ de la réalité filmée. En explorant l'invisible par les moyens du cinéma, et 
notamment par des procédés qui travaillent la durée et le temps (ralentis, accélérés, surimpressions, fondus, noirs, etc.), Gunvor Nelson propose d'expérimenter une autre réalité, celle du corps, qu'il soit collectif ou personnel, et de sa sensation. Le choc perceptif des images et des sons entre eux produit une compréhension, non pas discursive mais sensorielle du monde. Nous touchons au fond par le travail de la forme.

\section{ACTION-fiLMINg}

Dans les années quatre-vingt, Gunvor Nelson réalise une série de quatre films dits « de collage ", dans le cadre d'un atelier de création filmique à Stockholm - Frame Line (1983, 22', 16mm), Light Years (1987, 28', 16 mm), Light Years Expanding (1988, 25', $16 \mathrm{~mm})$, Field Study \#2 (1988, 8', 16 mm). Elle interroge son rapport à la fois joyeux et mélancolique à la Suède, pays natal qu'elle a quitté dans les années cinquante pour s'installer aux ÉtatsUnis. La cinéaste manipule et transforme la matière filmique en direct comme dans une sorte d'action-filming, utilisant différentes techniques de collage pour mettre en dialogue les couches d'images - à l'intérieur du plan mais aussi dans le film par un montage cut et des surimpressions. La technique du collage permet à la cinéaste de se libérer de la narration et du figuratif. Gunvor Nelson se focalise sur son plaisir matériel, explorant divers procédés pour couvrir, recouvrir, superposer les couches d'images. Elle peint en transparence sur une plaque en verre, dessine au crayon et colle des morceaux de papier dans l'image, tout cela en animation directe tandis qu'elle filme. Peu importe le support premier - peinture, photographie, carte postale, prise de vue réelle - car seul compte le processus de recouvrement. Faire et refaire, indéfiniment, de sorte que le matériau ne cesse de se transformer sous nos yeux. La cinéaste a conscience de son obsession du recommencement, revenant toujours sur certains motifs comme cette pomme qu'elle travaille de manière récurrente dans la série allant jusqu'à la laisser pourrir. Une pomme qui n'est pas sans rappeler la quête de Paul Cézanne mais aussi sa frustration et le délaissement de certaines œuvres demeurant, selon le peintre, inachevées.

D'une image à l'autre, Gunvor Nelson ne gomme pas les traces de ses travaux. Elle ne masque pas sa présence bord cadre. Au contraire, la cinéaste re-touche l'image dans l'image tandis que la performance artistique prend matériellement forme dans les films 
qui gardent la mémoire de leur mise en œuvre et véhiculent un peu de la personne qui les a engendrés.

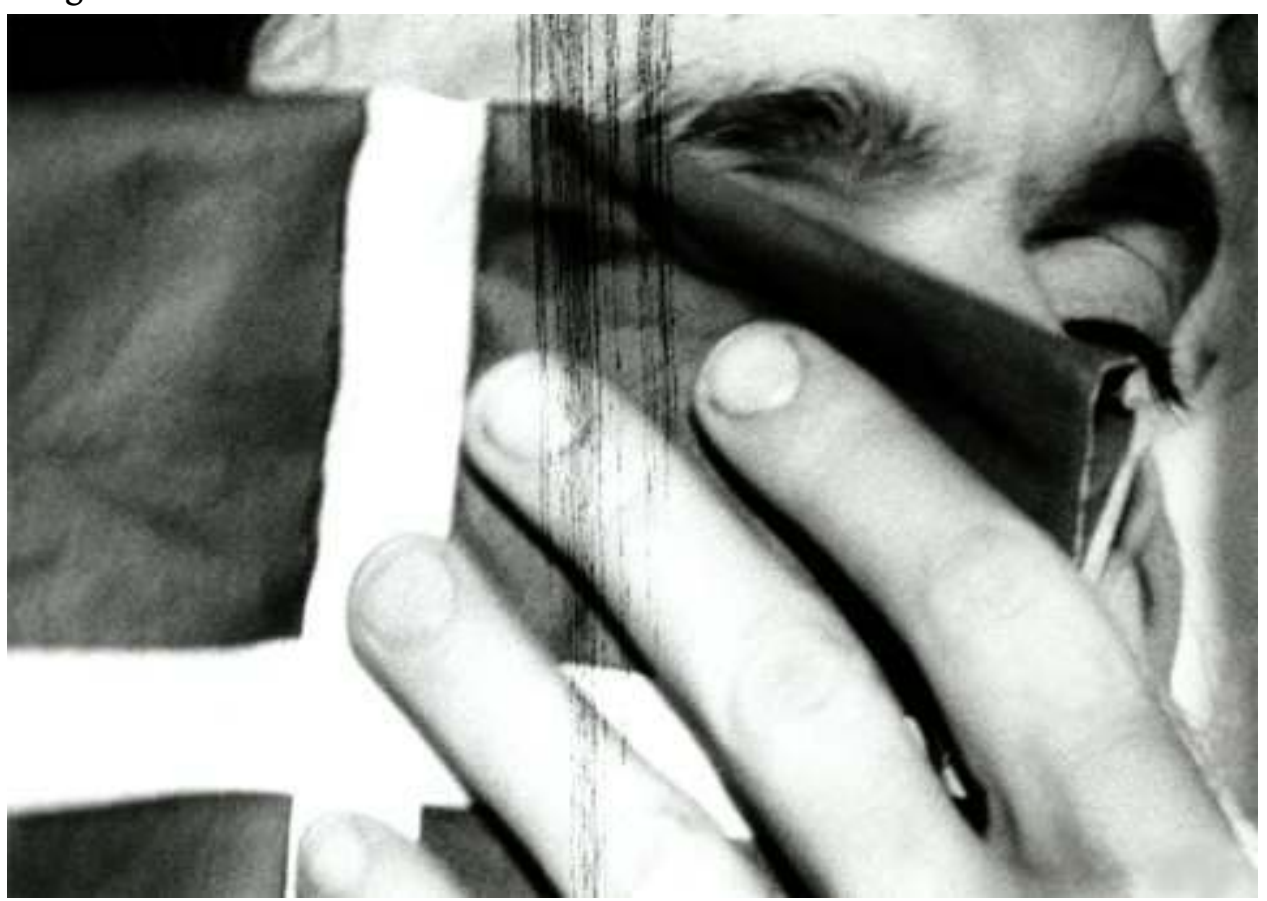

FIgURE 2 : FRAME LINE, 1983, 22'

Frame Line, le premier film de la série, s'ouvre in media res. Les mains de la cinéaste sont plongées dans une pâte blanche dont la texture lisse laisse penser qu'il s'agit de peinture. ${ }^{17}$ Sans doute est-ce une manière de rappeler dès l'incipit le programme haptique ici mis en œuvre : malaxer, pétrir, faire monter la pâte de la réalité par l'action d'un corps au travail. Ces mains réapparaîtront filmées en très gros plan à plusieurs reprises au cours du film mais à aucun moment nous ne verrons le corps de la cinéaste en entier. Il est saisi par morceaux (les mains, le visage et même un pied), selon le principe du montage. ${ }^{18} \mathrm{Il}$ n'y a donc pas de totalité première. Le corps de la cinéaste n'est pas représenté dans son ensemble, il est touché dans ses parties distinctes, perçu en quelque sorte de l'intérieur. Le spectateur fait l'expérience de ce corps réel, immédiat, un corps présenté et non pas représenté, un corps senti, perçu du dedans. Aucune vraisemblance n'est recherchée dans ce corps que la cinéaste veut d'abord nous faire éprouver. Car elle a pleinement conscience de la distance propre à la représentation. Lorsque le corps n'est pas isolé dans un geste ou une action (les mains au travail par exemple), il devient alors illusoire, irréél. Il s'agit en quelque sorte d'un corps fantôme, un corps dont l'image relève de l'apparition, brève mais insistante. Ainsi Gunvor Nelson entre à trois reprises dans le film, pausant quelques secondes en plan rapproché devant la caméra avant de disparaître. La cinéaste se montre en même temps qu'elle se dissimule : le visage flashé par la surexposition puis inversement masqué par de la peinture et un bonnet de laine noire - la peau est une surface sensible au même titre que la pellicule. Ce corps demeure in-visible, se dérobe à son enveloppe. Détaché du personnage et d'un possible romanesque, il a pris son indépendance, s'est vidé de sa substance «psychologique ». Nous avons la sensation de ce corps, nous le percevons à la fois comme un corps au travail et comme une matière à travailler, un corps éprouvé. Il est un matériau concret et son traitement plastique incarne bien l'expérience matérielle de la cinéaste. Le corps porte l'empreinte du projet et trouve naturellement place dans le grand collage animé du film. 

Expanding, troisième film de collage, s'ouvre cette fois sur un pinceau en action. Seuls la matière et le matériel de l'artiste sont visibles (caméra, pinceaux, palette, crayons, ciseaux). Le corps de l'artiste demeure hors champ. Le travail du matériau est mis en scène avec une distance maitrisée, à mi-chemin entre le trivial et le sacré de sorte que la matière semble animée d'une vie propre. La main du Pygmalion des premiers temps ${ }^{19}$ n'est plus nécessaire pour créer la vie. Le dessin se trace de lui-même, autonome, sans modèle. On retrouve ici le parti pris avant-gardiste d'une peinture en mouvement exprimé par Kandinsky et Leopold Survage puis reprise dans Le manifeste des peintres futuristes de 1910 : « (Le cinéma devra) parachever avant tout l'évolution de la peinture : se détacher de la réalité, de la photographie, du gracieux et du solennel. Devenir antigracieux, déformant, impressioniste, synthétique, dynamique, verbolibre. $»^{20}$ Dans ce cinéma, des tâches de peinture apparaissent et disparaissent sous nos yeux. Certains plans sont « bruités » (chants, cris, chuchotements, sifflets). Le montage est agité. Il règne un désordre foisonnant et ludique. Nous passons alors dans le film comme dans un labyrinthe. Notre œil s'égare le long des formes, se perd dans les coulures. Cette histoire manuelle que le film porte en lui demeure volontairement inachevée, suivant un art de la retouche permanente qui privilégie la résistance de cette matière que la cinéaste veut laisser agir par elle-même.

Si la matière vit et respire, ce film de collage n'en est pas moins habité par la figure de la main, invisible et pourtant sensible. Il est constitué des traces laissées par les mains absentes de la cinéaste comme en témoignent les manipulations explicites du matériau à la prise de vue et au montage. En quelque sorte, les mains sont ici négatives ${ }^{21}$, posées grandes ouvertes sur le film comme elles l'étaient déjà sur la pierre des grottes magdaléniennes de l'Europe Sud-Atlantique : seul leur contour enduit de couleur demeure. D'une image à l'autre, nous imaginons, nous fantasmons. Les coupes, les collures, les impuretés et les flous constituent les empreintes des mains de la cinéaste à l'œuvre et participent à la sensation de leur présence. Ces « marques accidentelles » ne concernent que la main du peintre écrit encore Gilles Deleuze à propos de Francis Bacon et elles « ne valent elles-mêmes que pour être utilisées, réutilisées par la main du peintre, qui va s'en servir pour arracher l'image visuelle au cliché naissant, pour s'arracher luimême à l'illustration et à la narration naissantes. Il va se servir des marques manuelles pour faire surgir la Figure de l'image visuelle. $»^{22}$ Le philosophe ajoute que le hasard est toujours acte et choix, qu'il n'est pas séparable d'une possibilité d'utilisation. Tout comme la peinture de Bacon ou de Cézanne, le cinéma de Gunvor Nelson est bien une addition d'images absentes ${ }^{23}$, une manipulation de hasards. Ces marques accidentelles de la main laissent place, dans l'imaginaire des spectateurs, au geste et à l'impulsion physique de la cinéaste, alors-même que l'image du corps est omise.

Dans cette série de films de collage, la conscience tactile s'exprime dans une relation déictique à l'image. Les empreintes physiques de la cinéaste sont autant de marqueurs spatiaux-temporels renvoyant à l'élaboration du film et la deixis devient en quelque sorte une attitude esthétique. Le corps de la cinéaste, à la fois visible et invisible, éprouvé et rêvé, a laissé les signes conscients de son passage. Comme si la cinéaste, ici et maintenant, travaillait encore à son achèvement dans une sorte de création-recherche où il s'agirait de penser le cinéma en même temps que de le faire. 


\section{L'imaginaire du matériau}

18 plus de trente ans, pour retrouver la Suède. C'est à cette période charnière que s'opère le
passage de la pellicule à la vidéo, portant l'artiste vers un autre type de sujet et de récit. Les possibilités de la vidéo conduisent Gunvor Nelson à explorer le corps imaginaire du matériau avec une indéfinissable liberté. La cinéaste n'échappe aucunement à la matière en abandonnant l'argentique. Elle explore une autre image, expérimente les procédés de sa restitution et son montage virtuel. Les transformations sont plus simples à réaliser, au tournage comme au montage, confie-t-elle. ${ }^{24}$ Gunvor Nelson rêve à la matière tel Francis Ponge, inspiré par les travaux de Gaston Bachelard, dans À la rêveuse matière..$^{25}$ L'opération consiste en une recherche purement matérielle qui va produire un nouvel espace plastique d'expression. La cinéaste tend à l'abstraction de la représentation, cherche à pénétrer la matière.

Dans ses premiers films tournés en vidéo, nous expérimentons le passage du concret à l'abstrait, traversant la réalité pour pénétrer progressivement la matière rêveuse. La pratique du collage est ici d'une autre nature. La caméra colle très concrètement à la réalité (très gros plans, arrêts sur image, absence de point et de focale) laquelle glisse paradoxalement de ce fait vers l'abstraction dans un drame de lignes, de couleurs et de rythmes. L'objet de la perception n'est plus séparable du sujet percevant et de son outil vidéo. La cinéaste ne reproduit pas le monde, elle le ressent, partant de ce qu'il y a de plus réel pour nous donner à percevoir quelque chose d'irréel. Car «Le cinéma, écrit Jean Epstein, est le plus puissant moyen de poésie, le plus réel moyen de l'irréel, du surréel, comme aurait dit Apollinaire. $»^{26}$

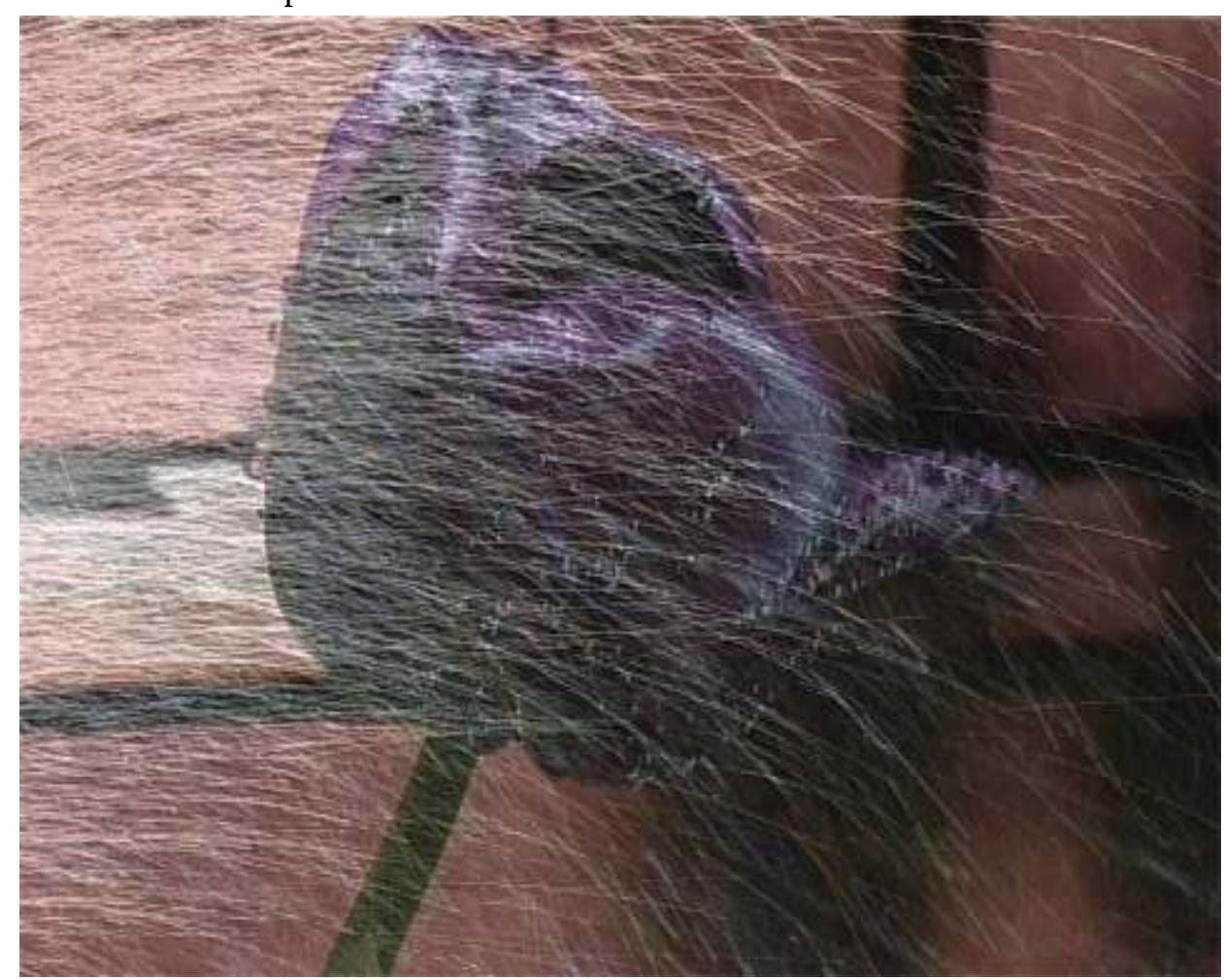

FIgURE 3 : TRUE TO LIFE, 2006, DV, 38' 

d'ingrédients, qui plus est prééxistants. Elle met en dialogue un train, les rails qui défilent (plans fournis avec le logiciel Première qu'elle apprend alors à utiliser) et la photographie d'un arbre. La creative method consiste à se laisser guider par le matériau au moment du tournage puis la cinéaste trouve un sens et se livre à l'expérimentation par un travail d'assemblage rigoureux et sensible au montage. Mais les règles ne sont pas fixées d'avance et le résultat est une aventure déroutante, à la fois physique et cérébrale. Le film oscille de manière instinctive entre le corps et l'esprit. Nous sommes dans le wagon en même temps que nos yeux perçoivent l'arbre par intermittence, entre deux battements de paupières. Puis l'alternance rapide des deux images - le train et l'arbre - nous transporte ailleurs, laissant place, comme le ferait une surimpression, à une troisième image qui s'exprime ici sous la forme de traits et de lignes. La vision culmine avec l'orage qui vient mettre un terme à cette expérience de déréalisation progressive. En travaillant contre ces éléments figuratifs que sont l'arbre et le train, Gunvor Nelson cherche à déconstruire le cliché du réel, à lui échapper pour retrouver une compréhension intuitive du monde.

Avec Snowdrift (a.k.a. Snowstorm, 2001, 9'), second projet vidéo, tout commence de la même manière dans le concret. Gunvor Nelson filme une tempête de neige dont elle va petit à petit pénétrer l'image au moyen de fondus et de ralentis, transformant cette matière première au montage par l'ajout de couleurs, d'éléments graphiques et d'effets numériques. Et la neige devient alors une pure image dont la texture vidéo brouille notre vision et notre perception réaliste du monde en nous transportant au cœur de cette tempête. Nos repères visuels et auditifs nous font défaut, nous découvrons de nouvelles sensations. Le glissement vers l'abstraction s'opère notamment par la tactilité de l'environnement sonore - mélange de bruits naturels, de sons électroniques, de mots chuchotés et de voix rauques - créant ainsi un niveau de compréhension inconnu, archaïque, à l'intérieur même de l'image. Nous parcourons le film comme un rêve intéractif où nous serions à la fois les spectateurs et les acteurs.

Dans True to Life (2006, 38', vidéo), Gunvor Nelson explore son petit jardin de Kristinehamn à ras du sol et le transforme en véritable terrain de jeu. La cinéaste progresse petit à petit dans le jardin au moyen de très gros plans jusqu'à toucher la matière végétale du bout de l'objectif. Rien n'échappe à la caméra qui traque le moindre détail. Tantôt Gunvor Nelson utilise la technique de l'image par image, joue sur l'exposition et le point, tantôt elle caresse un pistil, tandis que la main hors champ agite une branche. Le jardin s'anime comme par magie, semble vivant. Aux sons directs du tournage - toucher, marcher, filmer - s'ajoutent les bruits lointains de la maison et de la ville auxquels viennent se mêler des échos et des réverbérations travaillés au mixage. Les trois strates se confondent alors dans une étrange nappe sonore. Les insectes bourdonnent et l'orage gronde sur la jungle du jardin... Tout bouge à l'image comme au son, vit, foisonne, meurt dans une multitude de couches sonores et de formes graphiques. La plastique est en mouvement. Gunvor Nelson, dans le prolongement des avant-gardes historiques, construit des images mobiles ${ }^{27}$. C'est la forme du mouvement, la sensation du rythme que la cinéaste veut produire, et non reproduire, dans l'action réelle du tournage. L'image se creuse, opère une dissolution des formes et glisse progressivement vers l'image de la matière pure. Le signe devient signal : figures improvisées, camaïeux de sons et textures floutées dessinent un ailleurs qui soudain s'ouvre sur le ciel. Le jardin perd sa 
réalité, s'altère et devient presque effrayant. Cut au noir, ellipse, silence blanc. Il neige à présent.

L'approche de Gunvor Nelson est transformative. Le monde se métamorphose sous nos yeux par le biais d'un voyage matériel comme si la cinéaste cherchait à saisir une autre réalité dont la forme nouvelle se trouverait à l'intérieur même de la matière. L'exploration très concrète du matériau, celui de la réalité et du cinéma, se double d'une dimension abstraite laquelle pénètre progressivement le film. Cette dynamique alternant le concret et l'abstrait véhicule un questionnement esthétique simple portant sur la nature de l'image (et du son). Une image n'est pas juste une image, elle est forcément retravaillée, se révélant être une image qui pense et se réfléchit en tant qu'objet. La cinéaste manipule littéralement cette image en lui donnant une texture, de la profondeur et du relief, de sorte que celle-ci est traversée par un «flux $»^{28}$ flux de l'image et flux contenus dans l'image. Et la matérialité du film s'en trouve exaltée comme dans une vision mentale, mise en relation avec l'esprit d'où elle provient. Une conscience matérielle, habitée par des inspirations oniriques et impressionnistes.

Dans le «toucher-coller» de Gunvor Nelson, le rapport de l'œil et de la main est libre. Indépendante, la main n'est pas soumise à l'œil. La connexion entre les formes est manuelle avant d'être visuelle. Ce qui s'impose alors à la vue des spectateurs c'est un mouvement incessant, sans forme, c'est la «Figure» deleuzienne, la sensation pure. À propos de la peinture de Paul Cézanne et de Francis Bacon, Gilles Deleuze décrit la Figure comme une forme sensible rapportée à la sensation laquelle agit immédiatement sur le système nerveux qui est de la chair, ceci par opposition à la Forme abstraite qui agit quant à elle par l'intermédiaire du cerveau, plus proche de l'os. ${ }^{29}$

Peindre, ou filmer la sensation: telle est la ligne commune entre Paul Cézanne, Francis Bacon et Gunvor Nelson, aussi évidentes soient les différences entre leurs œuvres. Mais comment une peinture, ou un film, parviennent-ils à toucher le système nerveux et à procurer la sensation d'un corps, quel qu'il soit? C'est ce que nous avons tenté de mettre en lumière en nous attachant au rêve matériel de Gunvor Nelson et à la fonction génétique du " toucher-coller» dans son œuvre matérialiste ${ }^{30}$.

Si tous les cinéastes ont leur propre méthode de travail ${ }^{31}$, seuls certains tiennent à partager, dans un effort délibéré de la conscience, l'idée matérielle de l'œuvre. Ainsi, le cinéma de Gunvor Nelson n'est pas innocent, il cherche à se rapprocher de son immédiateté originelle, se maintenant là où le possible se joue, où le risque est absolu, comme s'il s'agissait de sa raison d'être en tant qu'œuvre. Ce cinéma libre et expérimental, a une conscience explicite de lui-même. Et cette tension, cette acuité propre, cette orientation totale vers l'œuvre à venir, constituent précisément le film qui se donne à voir. Il en résulte que les spectateurs ont la sensation physique et immédiate de ce rêve matériel. Le cinéma de Gunvor Nelson touche "à vue » et cherche à faire toucher par son élaboration sensible, ou par ce que nous proposons d'appeler une esthétique de la matérialité. 


\section{ANNEXES}

\section{FILMOGRAPHIE DE GUNVOR NELSON}

1966-1994 / Films 16 mm

Schmeerguntz, (Nelson \& Wiley), 1966, 15 min.

Fog Pumas, (Nelson \& Wiley), 1967, 25 min.

Kirsa Nicholina, 1969, $16 \mathrm{~min}$.

My Name is Oona, 1969, $10 \mathrm{~min}$.

Five Artists BillBobBillBillBob, (Nelson \& Wiley), 1971, 70 min.

Take off, 1972, $10 \mathrm{~min}$.

One \& The Same, (Nelson \& Freude), 1973, 4 min.

Moons Pool, 1973, 15 min.

Trollstenen, 1976, $120 \mathrm{~min}$.

Before Need, (Nelson \& Wiley), 1979, $75 \mathrm{~min}$.

Frame Line, 1983, $22 \mathrm{~min}$.

Red Shift, 1984, $50 \mathrm{~min}$.

Light Years, 1987, $28 \mathrm{~min}$.

Light Years Expanding, 1988, 25 min.

Field Study \#2, 1988, 8 min.

Natural Features, 1990, 30 min.

Time Being, 1991, 8 min.

Kristina's Harbor, 1993, 50 min.

Old Digs, 1993, 20 min.

Before Need Redressed, (Nelson \& Wiley), 1994, $42 \mathrm{~min}$.

1998-2012 / Vidéos, installations

Tree-Line, 1998, $8 \mathrm{~min}$.

Collected Evidence: 52 Weeks, 1999, installation.

Snowdrift (a.k.a. Snowstorm), 2001, 9 min.

Trace Elements, 2003, $10 \mathrm{~min}$.

True to Life, 2006, $38 \mathrm{~min}$.

New Evidence, 2006, 22 min.

Kristina Harbor's Revisited, 2010, 38 min. 
Alltintill NEAR, 2011, installation.

\section{NOTES}

1. «Je me contente de passer mes mains sur la surface des choses. " (Gunvor Nelson, in Gunvor Nelson and the Avant-Garde, ed. John Sundholm, 2003, p. 75. L'auteur traduit.)

2. Voir la filmographie de Gunvor Nelson à la fin de ce texte.

3. Je renvoie dans ce sens aux analyses de John Sundholm et de Steve Anker, tous deux spécialistes de son cinéma, ainsi qu'à l'ouvrage collectif cité précédemment, Gunvor Nelson and the Avant-Garde.

4. J'emprunte la formule au chapitre éponyme de Francis Ponge : «My creative method ", in Méthodes, Paris : Gallimard, 1961, p. 16.

5. « Renoncer, non pas à l'enquête, à l'investigation, à la préparation, aux repérages, à l'écriture préalable d'un scénario mais à l'avance que tout cela fait prendre sur le mouvement du film en train de se faire. Revenir au présent. À ce qui est en train de changer en même temps que nous et dont nous ne voyons pas le bout. Ne pas tout savoir, ne pas tout voir, n'avoir pas déjà tout vu... » (Jean-Louis Comolli, Voir et pouvoir, Paris : éd. Verdier, 2004, p. 167.)

6. Le terme « scénario » est confus car il peut aussi bien désigner le sujet ou l'histoire résumée en quelques lignes que la dynamique dramatique et l'enchaînement des situations ou encore, et c'est ici le sens auquel nous nous référons, un script abouti indiquant les dialogues, le jeu, le décor, les cadrages, les déplacements...

7. Selon l'hypothèse, discutable, de John Sundholm, cela s'expliquerait notamment par le fait qu'il s'agirait d'un « cinéma de femme » lequel n'imposerait pas un point de vue dominateur sur le monde filmé. La cinéaste ne chercherait pas à contrôler ou à juger comme pourrait le faire un cinéma masculin - aussi politiques soient ses films. Voir John Sundholm, The material and the mimetic, in Evidence, catalogue d'exposition, Karlstad, Suède, 2006, p. 30.

8. Le terme haptique vient du verbe grec aptô et signifie toucher. Selon Gilles Deleuze, il existe une qualité du regard située à la frontière du visible et du tactile. Ce regard haptique développe un espace lisse, un espace de proximité et d'affect intense, de contact. Dans Mille plateaux, le philosophe développe une opposition entre Espace lisse (haptique) / Espace strié (optique). L'haptique ou le lisse étant un espace de proximité, une forme de vision rapprochée, par opposition à un espace optique ou strié soit une forme de vision éloignée qui se déploierait dans un monde figé et articulé par des normes héritées de la perspective. (Gilles Deleuze, Félix Guattari, Mille plateaux, Paris : éd. de Minuit, 1980, pp. 614-622.)

9. «Enfin on parlera d'haptique [...] quand la vue elle-même découvrira en soi une focntion de toucher qui lui est propre, et n'appartient qu'à elle, distincte de sa fonction optique. On dirait alors que le peintre peint avec ses yeux, mais seulement en tant qu'il touche avec les yeux. Et sans doute, cette fonction haptique [...] peut aussi se recréer dans l'oeil moderne à partir de la violence et de l'insubordination manuelles. » (Gilles Deleuze, Francis Bacon. Logique de la sensation, Paris : éd. du Seuil, 2002, p. 146.)

10. La boîte verte donne rétrospectivement à voir, en 1934, les notes et les réflexions ayant servi à la genèse de La mariée mise à nu par ses célibataires, même (1915-1923). Elle a été confectionnée en 1934 (il existe un prototype de la Boîte qui date de 1914) et tirée à trois cents exemplaires par Marcel Duchamp. (Voir Duchamp du signe, Paris : éd. Flammarion, 1975.)

11. «Le propre de la pensée mythique, comme du bricolage sur le plan pratique, est d'élaborer des ensembles structurés, non pas directement avec d'autres ensembles structurés, mais en utilisant des résidus et des débris d'événements : odds and ends, dirait l'anglais, ou en français, des bribes et des morceaux, témoins fossiles de l'histoire d'un individu ou d'une société. En un sens, 
le rapport entre diachronie et synchronie est donc inversé : la pensée mythique, cette bricoleuse, élabore des structures en agençant des événements, ou plutôt des résidus d'événements, alors que la science, en marche du seul fait qu'elle instaure, crée, sous forme d'événements, ses moyens et ses résultats, ses hypothèses et ses théories. Mais ne nous y trompons pas : il ne s'agit pas de deux stades, ou de deux phases, de l'évolution du savoir, car les deux démarches sont également valides. » (Claude Lévi-Strauss, La pensée sauvage, Paris : éd. Plon, 1962, p. 36.)

12. On retrouve la figure du chiffonnier évoquée par Walter Benjamin comme métaphore de son activité d'historien qui n'amasserait que des vieux chiffons d'histoire: "Méthode de travail: montage littéraire. Je n'ai rien à dire. Seulement à montrer. Je ne vais rien dérober de précieux, ni m'approprier aucune formule spirituelle. Mais les haillons, les déchets, eux, je ne veux pas les inventorier, mais leur rendre justice de la seule façon possible : les utiliser. » (Paris, capitale du XIXème siècle, 1935, in Oeuvres, Paris : éd. Gallimard, 2000, p. 60.)

13. Gunvor Nelson prolongera cette réflexion sur le corps et l'image de la femme sous différentes formes dans son œuvre : le strip-tease métaphysique de Take off (1972, 10', $16 \mathrm{~mm})$, une naissance à la maison dans Kirsa Nicholina (1969, 16', 16 mm) ou encore Moons Pool (1973, 15', 16 mm), un essai sur le désir.

14. On retrouve ici la « disjonction voir/parler » évoquée par Gilles Deleuze à propos des cinémas de Syberberg, Straub et Duras : «On nous parle de quelque chose; on nous fait voir autre chose ; ce dont on nous parle est sous ce qu'on nous montre." (Gilles Deleuze, Qu'est-ce que l'acte de création ?, conférence filmée à la fémis par Arnaud des Pallières, 1987, 49'.)

15. Sur le caractère ontologique de l'image cinématographique, voir Stanley Cavell, La projection du monde. Réflexions sur l'ontologie du cinéma, Paris : Belin, 1999.

16. «J'appellerai photogénique tout aspect des choses, des êtres et des âmes qui accroît sa qualité morale par la reproduction cinématographique écrit Jean Epstein. [...] L'aspect photogénique d'un objet est la résultante de ses variations dans l'espace-temps. " L'ensemble des écrits cinématographiques de Jean Epstein a été publié en deux volumes par Pierre Lherminier : Écrits de cinéma, Paris : éd. Seghers, 1974.

17. Cette ouverture in media res rappelle aussi que l'artiste ne commence pas devant une toile blanche mais qu'au contraire il est habité par la réalité matérielle de sa pratique... Laquelle constitue déjà l'œuvre à venir.

18. «Dans l'acte de filmer un plan ou celui de sculpter une main, il n'y a pas soustraction ou division ; c'est la partie qui est déjà totalité. Sans doute cette main entretient-elle par ailleurs un rapport avec d'autres fragments, ou d'autres totalités, mais ces rapports sont plus forts de n'être pas imposés. Penser le corps dans sa vérité, c'est ainsi peut-être le penser dans des rapports inédits, des correspondances qui échappent à la matérialité de la silhouette. Le rapport que nous entretenons à notre corps est lui-même le fruit d'un montage ; il est logique que le montage nous en rende l'image. » (Vincent Amiel, Le corps au cinéma. Keaton, Bresson, Cassavetes, Paris: PUF, 1998, p. 52.)

19. Dès 1908, Émile Cohl introduisait ses mains dans Fantasmagorie pour dévoiler la naissance de sa créature en trois coups de crayon.

20. Repris in Dominique Noguez, Cinéma, théorie, lectures, numéro spécial de la Revue d'esthétique, Paris : éd. Klincksieck, 1973.

21. En 1979, Marguerite Duras re-monte deux films, Césarée et Les mains négatives, à partir des rushes du Navire Night, Paris filmé à l'aube devient, sous l'action de la voix off, une grotte noire et bleue habitée par les balayeurs et les femmes de ménage du petit matin : «Devant l'océan/ sous la falaise/ sur la paroi de granit// ces mains// ouvertes// Bleues/ Et noires// Du bleu de l'eau/ Du noir de la nuit ».

22. Gilles Deleuze, Francis Bacon. Logique de la sensation, op. cit., p. 89. 
23. «Le film est moins une soustraction de ses images réelles qu'une addition de ses images absentes. " (Marcel Hanoun, in Cinéma cinéaste. Notes sur l'image écrite, Crisnée, Yellow Now, 2001, p. 82.)

24. «[...] il y a des différences. La pellicule est plus subtile. Bien sûr, j’apprécie l'immédiateté de la vidéo. Certains aspects techniques sont plus faciles à réaliser comme les surimpressions qui sont particulièrement laborieuses en pellicule. Il faut délimiter le segment exact à l'attention du laboratoire et décrire comment il devra être retiré. En vidéo, par contre, on peut manipuler les images soi-même et laisser son ordinateur faire le travail. [...] Mon approche du matériau, ma façon de filmer et de monter reste identique pour les deux supports. En vidéo comme en pellicule, j'essaye d'explorer de nouveaux territoires, parce que chaque projet induit une solution, une démarche qui lui est propre. C'est la technique qui diffère. Quand j'ai commencé à travailler en vidéo, j'ai dû me familiariser avec ce nouveau medium et apprendre à le maîtriser. » (Conversation avec Gunvor Nelson, par Julie Savelli, in Bref le magazine du court-métrage, $\mathrm{n}^{\circ}$ 102, maijuin 2012, p. 24. L'auteur traduit.)

25. Le poète compare la « Nature entière » à une écriture et affirme qu'il suffit de «nommer quoi que ce soit " pour " glorifier la matière » et « exprimer tout de l'homme ». (Francis Ponge, " À la rêveuse matière » (1962), in Cuvres complètes, t. I, p. 869.)

26. Jean Epstein, in Le cinématographe vu de l'Etna, Paris : Les Écrivains réunis, 1926.

27. Dans le texte sur son film Le Ballet mécanique (1924), Fernand Léger écrit : "Construire des films sans scénario en considérant l'image mobile comme personnage pricnipal. »

28. Sur cette question, je renvoie au très bel ouvrage de Didier Coureau, Flux cinématographiques, Cinématographie des flux paru en 2010 chez L'Harmattan.

29. "La sensation c'est le contraire du facile et du tout fait, du cliché mais aussi du sensationnel, du spontané, etc. La sensation a une face tournée vers le sujet (le système nerveux, le mouvement vital, l'instinct, le tempérament, tout un vocabulaire commun au Naturalisme et à Cézanne), et une face tournée vers l'objet (le fait, le lieu, l'événement). [...] à la fois je deviens dans la sensation et quelque chose m'arrive par la sensation, l'un par l'autre, l'un dans l'autre. Et à la limite c'est le même corps qui la donne et qui la reçoit, qui est à la fois objet et sujet. Moi spectateur, je n'éprouve la sensation qu'en entrant dans le tableau, en accédant à l'unité du sentant et du senti. [...] La couleur est dans le corps, la sensation est dans le corps, et non dans les airs. [...] Ce qui est peint dans le tableau c'est le corps, non pas en tant qu'il est représenté comme objet, mais en tant qu'il est vécu comme éprouvant telle sensation (ce que Lawrence, parlant de Cézanne, appelait "l'être pommesque de la pomme"). [...] Selon un mot de Valéry, la sensation c'est ce qui arrive directement, en évitant le détour ou l'ennui d'une histoire à raconter. » Gilles Deleuze, Francis Bacon. Logique de la sensation, op. cit., p. 39-50.

30. «Un film matérialiste est un film qui ne donne pas du réel des reflets illusoires, qui ne donne pas de reflets du tout, mais partant de sa propre matérialité (écran plat, pente idéologique, naturelle, specttaeurs) et de celle du monde, les donne à voir dans un même mouvement... Mais... encore faut-il qu'il soit dialectique... Un film dialectique, c'est donc un film qui se déroule... en faisant savoir par quels procès de transformation... une connaissance ou une représentation devient matière écranique et par quels autres procès cette matière filmique se transforme en connaissance et en représentation chez le spectateur." (Cinéthique, ${ }^{\circ}{ }^{\circ}$, p. 21, cité par Daniel Serceau et Joël Magny dans CinémAction, «Histoire des théories du cinéma », n60, La querelle de l'impression de réalité, 1991, p. 113.)

31. "L'honnête homme a son propre code dans le sang, le génie se fait en vivant son propre code. Oui, oui, le génie qui n'ignore rien des autres se fait sa propre méthode. » Propos de Paul Cézanne rapportés dans Cézanne, co-réalisation Danièle Huillet et Jean-Marie Straub, 1989, à partir du dialogue avec Joachim Gasquet (éd. Berheim - Jeune). 


\section{RÉSUMÉS}

Comment une peinture, ou un film, parviennent-ils à toucher le système nerveux et à procurer la sensation d'un corps, quel qu'il soit? C'est ce que nous souhaitons mettre en lumière en nous attachant au rêve matériel de Gunvor Nelson et à la fonction génétique du "toucher-coller " dans son œuvre matérialiste. Si tous les cinéastes ont leur propre méthode de travail, seuls certains tiennent à partager, dans un effort délibéré de la conscience, l'idée matérielle de l'œuvre. Ainsi, le cinéma de Gunvor Nelson n'est pas innocent, il cherche à se rapprocher de son immédiateté originelle, se maintenant là où le possible se joue comme s'il s'agissait de sa raison d'être en tant qu'œuvre. Ce cinéma libre et expérimental a une conscience explicite de lui-même. Et cette tension, cette orientation totale vers l'œuvre à venir, constituent précisément le film qui se donne à voir. Il en résulte que les spectateurs ont la sensation physique et immédiate de ce rêve matériel. Le cinéma de Gunvor Nelson touche "à vue » et cherche à faire toucher par son élaboration sensible, ou par ce que nous proposons d'appeler une esthétique de la matérialité.

Dream and Material. Touch and Paste in Gunvor Nelson's cinema

How do a painting or a film manage to touch the nervous system and give the sensation of a body, whatever it is? This is what we hope to throw light on by focussing on Gunvor Nelson's material dream and on the genetic function of the "touch and paste" in her material work. All film-makers have their own working methods but few of them really wish to share, in a deliberate effort of self- conscience, the material conception of the work. In that way, Gunvor Nelson's cinema is not innocent, it tries to get closer to its creative source, staying on a border line where what's possible is at stake as if it was its reason for being as a work of art. This free experimental cinema has an explicit conscience of itself. And this tension, this total dedication to the work that is to be born, precisely make up the film we are watching. As a result the spectators have the immediate physical sensation of this material dream. Gunvor Nelson's cinema touches the material under our eyes and tries to make us touch it through its sensitive elaboration or through what we suggest to call an aesthetics of materiality.

\section{AUTEUR}

\section{JULIE SAVELLI}

PAST Cinéma à l'université Paul Valéry - Montpellier 3, responsable du Master 2 professionnel «Direction artistique de projets culturels ». Membre du laboratoire Rirra21, ses recherches portent sur la conscience créatrice, dans la continuité de sa thèse de doctorat soutenue en 2007 à l'université Toulouse Le Mirail sous la direction de Guy Chapouillé. Julie Savelli a également diverses activités professionnelles dans les domaines de l'édition et de la programmation documentaire. 